\title{
Functions of chromatin remodeling factors in heterochromatin formation and maintenance
}

\author{
BI Xin \\ Department of Biology, University of Rochester, Rochester, NY 14627, USA \\ Received October 26, 2011; accepted December 4, 2011
}

\begin{abstract}
Heterochromatin is characteristically more compact than euchromatin in the eukaryotic genome. The establishment of heterochromatin is mediated by special histone modifications, recruitment and propagation of heterochromatin specific proteins, as well as formation of special primary and high order structures of chromatin. Chromatin remodeling factors are ATPases that can alter the conformation and/or positioning of nucleosomes along DNA in an ATP-dependent manner. There is increasing evidence implicating chromatin remodeling activities in heterochromatin in various organisms ranging from yeasts to humans. Chromatin remodeling factors play roles in the establishment, maintenance and epigenetic inheritance of heterochromatin, but the underlying molecular mechanisms have just begun to be investigated.
\end{abstract}

chromatin remodeling, gene silencing, heterochromatin, inheritance, nucleosome, replication

Citation: Bi X. Functions of chromatin remodeling factors in heterochromatin formation and maintenance. Sci China Life Sci, 2012, 55: 89-96, doi: $10.1007 / \mathrm{s} 11427-012-4267-1$

\section{Heterochromatin and chromatin remodeling factors}

Eukaryotic DNA is packaged into chromatin through the formation of nucleosomes. Each nucleosome consists of 147 bp of DNA wrapped around a protein core made of two copies of each of histones H2A, H2B, H3 and H4 [1]. There are two general types of chromatin interspersed in the genome that are structurally and functionally different. One is euchromatin that is decondensed and generally accessible to factors involved in DNA transactions. The other is heterochromatin that is condensed and mostly refractory to DNA transaction factors $[2,3]$. Heterochromatin formed at selected loci plays important roles in genome stability and epigenetic regulation of genome activities, which impacts cell proliferation and differentiation, organismal development and aging [4].

email: xin.bi@ rochester.edu
The formation and maintenance of heterochromatin in different organisms follow a common theme, but the molecular details differ between species [2,5]. Heterochromatin has characteristic histone modifications that are recognized by repressor/silencing complexes that serve as an integral part of heterochromatin. In fission yeast and higher eukaryotes, histones in heterochromatin are hypoacetylated and selectively methylated at lysine 9 of histone H3 (H3-K9) [2]. A major heterochromatin associated protein, HP1, preferentially binds tri-methylated H3-K9 [2]. In the budding yeast Saccharomyces cerevisiae, histones in heterochromatin are hypoacetylated in general, but are not methylated at $\mathrm{H} 3-\mathrm{K} 9$, and the SIR complex preferentially associates with hypoacetylated nucleosomes in heterochromatin [5]. Heterochromatin is also characterized by the high regularity of the positioning/phasing of the nucleosomes in it, which is believed to facilitate the folding of chromatin into high order structures $[2,3,6,7]$. Formation of heterochromatin is mediated by the recruitment and association/propagation of silencing 
complexes along chromatin. A silencing complex typically consists of one or more enzymes that carry out heterochromatin specific modification(s) of histones and other component(s) that recognize these modification(s).

Chromatin remodeling enzymes are SNF2 family ATPases that use the energy of ATP hydrolysis to mobilize or rearrange nucleosomes in chromatin [8]. All eukaryotes have multiple chromatin remodeling enzymes that can restructure, move, or eject nucleosomes, thereby regulating the dynamic properties of chromatin. Chromatin remodeling activities participate in genomic transactions including transcription, DNA repair and recombination, as well as chromatin assembly [8]. Based on the possession of specific conserved motifs, the SNF family proteins can be divided into many subgroups including the SWI/SNF, ISW, Mi-2/CHD and the FUN30/ETL1 subfamilies [8]. The high regularity of nucleosomes in heterochromatin may be achieved during chromatin replication when new nucleosomes are formed. Alternatively, or in addition, preexisting nucleosomes may be repositioned to form ordered arrays. There is increasing evidence suggesting an involvement of chromatin remodeling in the formation and maintenance of heterochromatin in organisms ranging from yeasts to humans (Table 1).

\section{Roles of members of the FUN30/ETL1 sub- family of chromatin remodelers in heterochro- matin}

The FUN30/ETL1 subgroup of SNF2 family proteins is found in all eukaryotes except the lowest ones $[9,10]$. They include Fun 30 in $S$. cerevisiae, Fft1, Fft 2 and Fft3 (Fission yeast Fun Thirty) in the fission yeast Schizosaccharomyces pombe, Etl1 (Enhancer Trap Locus 1) in mice, and SMARCAD1 (SMARCA containing DEAD box 1) in hu-

Table 1 Chromatin remodeling factors linked to heterochromatin

\begin{tabular}{cccc}
\hline Name & Organism & Category & References \\
\hline Fun30 & S. cerevisiae & FUN30/ETL1 & {$[10,11]$} \\
Fft3 & S. pombe & FUN30/ETL1 & {$[12]$} \\
SMARCAD1 & Mammals & FUN30/ETL1 & {$[13]$} \\
& & & \\
Isw1 & S. cerevisiae & ISW & {$[11,14]$} \\
NURF & Drosophila & ISW & {$[15,16]$} \\
WICH & Mammals & ISW & {$[17-19]$} \\
ACF1-SNF2H & Mammals & ISW & {$[20]$} \\
& & & \\
Mi-2/NuRD & Human & CHD & {$[21,22]$} \\
Mit1 & S. pombe & CHD & {$[23,24]$} \\
& & & {$[25-31]$} \\
ATRX & Mammals & SWI/SNF & {$[32,33]$} \\
dATRX & Drosophila & SWI/SNF & {$[34]$} \\
BRG1 & Human & SWI/SNF &
\end{tabular}

mans, and have shared features within the common helicase-like region. The biochemical properties of this group of proteins have not been extensively studied. Interestingly, three members of this group, Fun30, Fft3 and SMARCAD1 have been shown to function in the formation and/or maintenance of heterochromatin in their respective host organisms.

\subsection{Fun30}

S. cerevisiae has long been used as a model for studying heterochromatin structure and function. Heterochromatin in $S$. cerevisiae exists at the cryptic mating loci $H M L$ and $H M R$, as well as subtelomeric regions [5]. Histones in heterochromatin are hypoacetylated and hypomethylated $[5,35,36]$. The SIR silencing complex consisting of Sir2, Sir3 and Sir4 preferentially binds hypoacetylated nucleosomes, and serves as an integral part of heterochromatin [5]. Removal of any of the three Sir proteins leads to a complete disruption of heterochromatin. Sir2 is a conserved protein deacetylase that carries out the hypoacetylation of histones in heterochromatin [37]. Formation of heterochromatin is initiated at cis-acting silencers flanking the $H M$ loci or telomeric repeats that associate with proteins capable of recruiting the SIR complex [5] (Figure 1A and B). The SIR complex deacetylates histones in nearby nucleosomes, thereby creating high affinity binding sites for recruiting additional SIR complexes (Figure 1B and C). Through repeated cycles of histone deacetylation and SIR complex

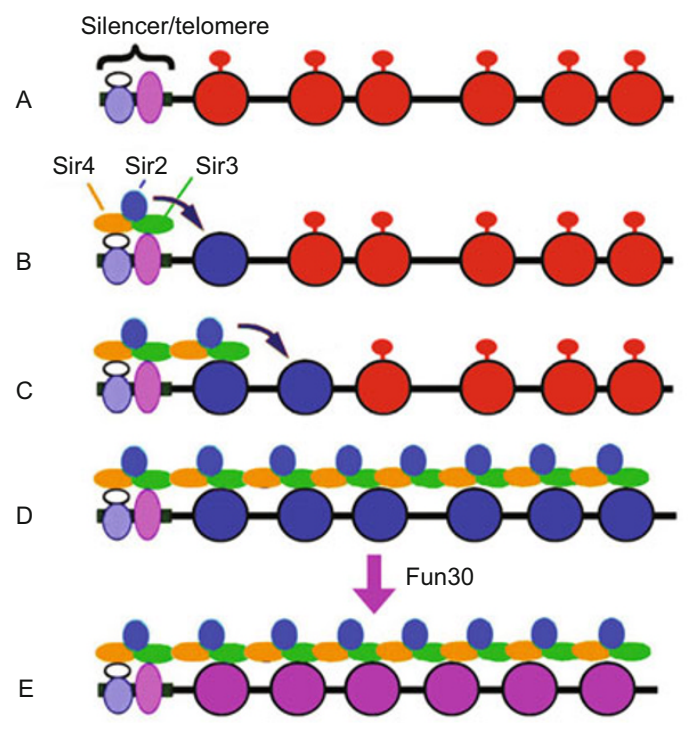

Figure 1 A model for the role of Fun30 in the formation of heterochromatin in S. cerevisiae. A-D illustrate the propagation of SIR complex during the formation of $S$. cerevisiae heterochromatin. A, A silencer or telomere recruits proteins that in turn recruit the SIR complex. B, Sir2 in the SIR complex deacetylates a nearby nucleosome. C, The hypoacetylated nucleosome recruits an incoming SIR complex. D, Repeated cycles of histone deacetylation and SIR complex recruitment lead to the spread of SIR complex. E, Fun30 acts to rearrangement chromatin associated with SIR complexes, resulting in fully silenced, mature heterochromatin. 
recruitment, SIR complexes propagate along the chromatin to form a heterochromatin domain [5] (Figure 1C and D).

The primary chromatin structure pertaining to nucleosome positioning/phasing at each of the $H M$ or subtelomeric loci is markedly different when it is in a heterochromatic state than when it is in a derepressed state (e.g., when any of the Sir proteins is absent) [38-40]. Therefore, formation of heterochromatin involves significant rearrangement of nucleosomes. However, none of the Sir proteins has chromatin remodeling activity. There is evidence for the involvement of Fun30 in the formation of heterochromatin structure. Fun30 was recently shown to have nucleosome sliding and histone $\mathrm{H} 2 \mathrm{~A} / \mathrm{H} 2 \mathrm{~B}$ dimer exchange activities [41]. It is required for efficient transcriptional silencing at the $H M$ and telomeric loci, and is enriched at these loci $[10,11]$. In the absence of Fun30, only a partially silenced chromatin structure is established at the $H M L$ locus. Such a structure resembles fully silenced heterochromatin in histone modifications and the association of SIR complex, but differs markedly from both fully silenced and derepressed chromatin structures regarding nucleosome positioning [11]. Moreover, without Fun30, de novo establishment of heterochromatin could only reach an intermediate structure [11]. These studies suggest that Fun30 serves to convert an intermediate structure to mature heterochromatin structure during the formation of heterochromatin.

A possible mechanism for the function of Fun30 in heterochromatin is that it acts to create a chromatin configuration prior to heterochromatin formation that is favorable for the spreading of SIR complex. This model predicts that Fun30 modulates $H M L$ chromatin in the absence of SIR complex. However, this was found not to be the case [11], which argues against the notion that Fun30 modulates derepressed chromatin in preparation for SIR complex spreading. As such, Fun30 most likely remodels/repositions nucleosomes, simultaneously with, or after, the spreading of SIR complex along chromatin. Fun30 may remodel a nucleosome immediately after it is deacetylated by SIR complex, or vice versa (i.e., SIR complex deacetylates and binds the nucleosome immediately after it is remodeled by Fun30). Alternatively, Fun30 may remodel chromatin after SIR complex has spread across the silent regions. This model implies that the spreading of SIR complex per se only results in the formation of an immature/intermediate state of heterochromatin that is converted by Fun30 to mature, fully silenced, heterochromatin (Figure 1D and E).

The fact that fun30 $\Delta$ alters heterochromatin but not derepressed chromatin at $H M L$ suggests that Fun30 function is selectively directed to heterochromatin [11]. What then is the mechanism for this specificity? Many chromatin remodeling proteins associate with other factors to form multi-subunit complexes that contain conserved motifs such as the SANT, bromodomain and chromodomain that recognize distinct features of chromatin and aid in the targeting of the remodeling activities to specific chromatin structures/loci
[8]. However, two independent biochemical studies aimed at finding potential Fun30-interacting factors failed to yield any $[10,41]$. Instead, it was found that Fun30 forms a homodimer [41]. On the other hand, Fun30 (and other FUN30/ ETL1 members) per se possesses a CUE (Coupling of Ubiquitin conjugation to ER degradation) motif that is similar to the UBA (Ubiquitin Associated domain) motif that binds ubiquitin $[10,42]$. The putative CUE motif of Fun30 is required for its full function in $H M R$ silencing [10]. It is tempting to posit that the CUE motif of Fun30 helps to target Fun30 activity to heterochromatin by recognizing a ubiquitinated component(s) of heterochromatin.

\subsection{Fft3}

In the fission yeast $S$. pombe and higher eukaryotes, heterochromatin is characterized by histone deacetylation and H3-K9 methylation that is recognized by various homologs of heterochromatin protein 1 (HP1) [2]. Fission yeast heterochromatin exists at the pericentric, mating type, telomeric and rDNA loci, and is associated with the HP1 ortholog Swi6. Deletion of Fft 3 causes alterations in the structure of pericentric heterochromatin as probed by MNase cleavage [20]. On the other hand, the abundance of histone $\mathrm{H} 3$ or the methylation of $\mathrm{H} 3-\mathrm{K} 9$ within heterochromatin is not affected. Whether fft $3 \Delta$ affects nucleosome arrangement and/or Swi6 binding at heterochromatin has not been examined. The special centric chromatin containing CENP-A instead of histone $\mathrm{H} 3$ is also affected by $f f t 3 \Delta$, as evidenced by the decrease in CENP-A level and concomitant increase in the levels of histone $\mathrm{H} 3$ as well as acetylation of histones H3 and H4, suggesting euchromatinization of this region [12]. In contrast to Fun 30 in budding yeast, Fft 3 is not enriched at heterochromatin per se, but is instead associated with boundary/barrier elements demarcating pericentric and subtelomeric heterochromatin domains [12]. It was proposed that $\mathrm{Fft} 3$ acts at the boundaries to protect centromeric and subtelomeric domains from euchromatin formation [12]. This is consistent with previous findings that insulators assume special chromatin structures, and the functions of certain insulators depend on chromatin remodeling activities [43-45]. As there is evidence suggesting a role of nucleosome exclusion in the functions of heterochromatin boundary elements [46,47], it is possible that Fft3 helps to evict nucleosomes at the boundaries. However, whether Fft3 has chromatin remodeling activity has not been tested.

\subsection{SMARCAD1}

The inheritance of chromatin state, or epigenetic information, is believed to occur via a self-template mechanism in which intact or split parental nucleosomes are distributed randomly to the two nascent DNA strands at the replication fork $[48,49]$. Nucleosomes containing new histones are positioned among the old ones, and are modified by chromatin 
modifying enzymes bound to old histones/nucleosomes. As a consequence, the state of nascent chromatin is converted to that of parental chromatin. In this model, the chromatin state is only partially disrupted by DNA replication, and is subsequently re-established.

A recent report found human SMARCAD1 to play a role in the maintenance/re-establishment of heterochromatin during DNA replication [13]. The ATPase activity of SMARCAD1 was found to be necessary for global deacetylation of histones $\mathrm{H} 3 / \mathrm{H} 4, \mathrm{H} 3-\mathrm{K} 9$ methylation and the establishment of heterochromatin. SMARCAD1 associates with transcriptional repressors including KAP1, histone deacetylases HDAC1/2 and the histone methyltransferase G9a/GLP, and modulates the interaction of HDAC1 and KAP1 with heterochromatin. In addition, SMARCAD1 directly interacts with PCNA, a component of the replication machinery, and is recruited to sites of DNA replication (Figure 2A).

Histones $\mathrm{H} 3$ and $\mathrm{H} 4$ deposited on newly replicated DNA are highly acetylated. When heterochromatin loci are replicated, the acetylation of newly deposited histones has to be removed before further heterochromatin-specific modifications could take place. SMARCAD1 is believed to remodel nascent nucleosomes, thereby facilitating the deacetylation of newly deposited histones in a replication-coupled manner, and aiding in the inheritance of heterochromatin during DNA replication (Figure 2). However, SMARCAD1 has not been demonstrated to have chromatin remodeling activity in vitro [13]. As such, how SMARCAD1 facilitates the deacetylation of histones has yet to be determined.

\section{Functions of the ISW family of chromatin remodelers in heterochromatin}

The ISWI (imitation switch) family remodelers include Isw1 and Isw2 in budding yeast, dNURF, dCHRAC and dACF in Drosophila, and hWICH and hNoRC in humans
$[8,50]$. A characteristic feature of ISW proteins is a C-terminal pair of SANT (SWI3, ADA2, hNCoR, hTFIIIB) and SLIDE (SANT-like ISWI domain) domains that constitutes a nucleosome recognition module. Many ISWI proteins can act to modulate nucleosome spacing thereby facilitating chromatin assembly or transcriptional repression. On the other hand, some complexes (e.g., NURF) can randomize nucleosome positioning to help transcription by RNA polymerase II. There is evidence linking several ISWI members to heterochromatin.

\subsection{Isw1}

In $S$. cerevisiae, ISW1, similar to FUN30, is also required for $H M L$ and $H M R$ silencing [11,14]. However, unlike FUN30 deletion, ISWI deletion does not significantly affect the overall state or primary structure of heterochromatin [11]. ISW1 deletion also does not affect de novo establishment of $H M L$ heterochromatin. Instead, isw $1 \Delta$ significantly reduces the stability of $H M L$ heterochromatin [11]. Therefore, while Isw 1 is not required for the formation of heterochromatin, it is critically required for maintaining the stability of $H M L$ heterochromatin structure. This is in line with the fact that Isw1 has the ability to stabilize nucleosomes [51]. The ISWI complex in Drosophila regulates high-order chromatin structure [52]. Isw1 might also help to maintain yeast heterochromatin by stabilizing high-order chromatin structures.

\subsection{NURF}

Drosophila NURF has nucleosome sliding activity and helps transcription of chromatin template in vitro, and is required for transcription activation in vivo [53]. NURF was recently found to associate with HP2, an HP1 homolog known to associate with HP1 and be involved in heterochromatin-mediated gene silencing [15]. Moreover, NURF also interacts with Nap-1 (nucleosome assembly protein 1),

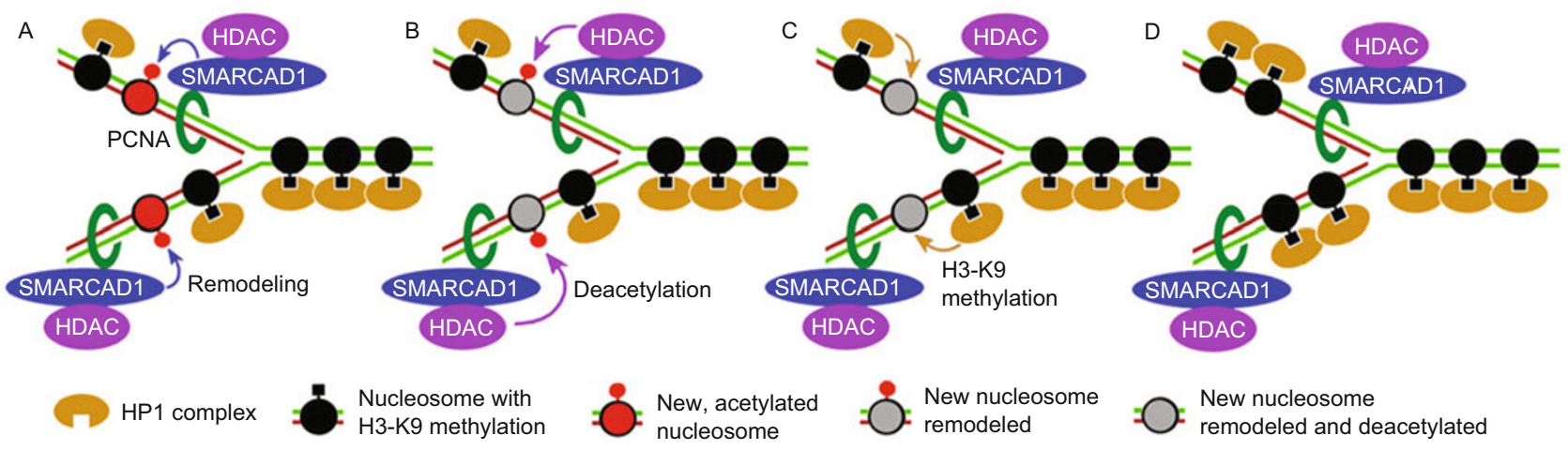

Figure 2 Model for the function of SMARCAD1 in the inheritance of heterochromatin in mammalian cells. A, SMARCAD1 in complex with HDAC is recruited to the replication fork via its association with PCNA, and remodels newly formed, acetylated nucleosomes. B, HDAC associated with SMARCAD1 deacetylates nascent nucleosomes after they are remodeled by SMARCAD1. C, The silencing complex (HP1 complex) associated with old nucleosomes methylates histone H3-K9 in the remolded and deacetylated nascent nucleosomes. D, Nascent nucleosomes methylated at H3-K9 recruit HP1 complexes. Note processes $\mathrm{C}$ and $\mathrm{D}$ may occur in a co-replicational or post-replicational manner. 
a histone $\mathrm{H} 2 \mathrm{~A} / \mathrm{H} 2 \mathrm{~B}$ chaperone required for heterochromatin-mediated position effect variegation [15,16]. These findings suggest that NURF may cooperate with HP2 and Nap-1 to contribute to the formation of heterochromatin.

\subsection{WICH}

WICH (WSTF-ISWI chromatin remodeling complex) is a conserved vertebrate chromatin remodeling complex that is composed of ISWI type chromatin remodeler SNF2H and WSTF (Williams Syndrome Transcription Factor). Both mouse and frog WICHs are able to assemble regularly spaced nucleosome arrays in vitro [17]. In human cells, WSTF was found in pericentric heterochromatin during its replication, suggesting a role for WSTF (and by inference WICH) in the replication of heterochromatin [17]. It was further demonstrated that WICH is targeted to replication foci via the interaction between WSTF and PCNA [18]. Removal of WSTF renders newly replicated chromatin more refractory to DNase I, suggesting that WICH is involved in maintaining an "open" chromatin structure after replication [18]. Such an "open" configuration is proposed to provide a window for chromatin modifying enzymes and chromatin binding factors to access nascent chromatin and reestablish the proper epigenetic state of chromatin [19]. Without this window, chromatin fiber is believed to form prematurely, leading to excessive heterochromatin formation [19].

\subsection{ACF1-SNF2H}

ACF1 (ATP-utilizing chromatin assembly and remodeling factor 1) and SNF2H form a complex that is also specifically enriched in replicating pericentric heterochromatin [20]. Knockdown of mammalian ACF1 specifically impairs the replication of pericentric heterochromatin, and induces a delay in cell-cycle progression through late $S$ phase in which heterochromatin is replicated. Mutational disruption of ACF1-SNF2H interaction also interferes with replication of condensed chromatin. These data suggest that the ACF1-SNF2H complex is involved in a mechanism that enables DNA replication through condensed heterochromatin [20].

\section{Functions of the CHD family of chromatin remodeling factors in heterochromatin}

The CHD (chromodomain helicase DNA-binding) family chromatin remodelers each possess two N-terminal chromodomains that can interact with other factors [8]. CHD proteins are transcriptional regulators that are important for development. Certain CHD complexes promote transcription by repositioning or removing nucleosomes. On the other hand, other CHD complexes such as vertebrate $\mathrm{Mi}-2$ /
NuRD (nucleosome remodeling and deacetylase) complex play a negative role in transcription.

\subsection{Mi-2/NuRD}

In human T cells, Mi-2 and HDAC form a complex with Ikaros, a DNA-binding protein required for lineage determination and proliferation control in the lymphoid system [21]. Such an Ikaros-NuRD complex has chromatin remodeling and histone deacetylation activities in vitro. When a $\mathrm{T}$ cell is activated, Ikaros recruits Mi-2/HDACs to heterochromatin loci. It is believed that Mi-2/NuRD complex is involved in a mechanism for replicating heterochromatin specific to lymphoid cells that undergo rapid proliferation [22].

\subsection{Mit1}

Fission yeast Mit1 (Mi-2-like protein interacting with $\mathrm{Clr}$ three 1 ) is highly similar to Mi-2 proteins but lacks a chromodomain. It was identified as a component of the multienzyme SHREC-2 silencing complex that contains Clr1, Clr2, Clr3, Mit1 and Chp2 [23,24]. SHREC-2 mediates heterochromatic gene silencing and is distributed throughout all major heterochromatin domains [23]. Given that Clr3 is a histone deacetylase, and that Chp2, an HP1 homolog, recognizes methylated histone $\mathrm{H} 3-\mathrm{K} 9$, the SHREC-2 complex links H3-K9 methylation and histone deacetylation involved in heterochromatin formation [24]. Mutational inactivation of Clr3 and Mit1 results in changes in the primary heterochromatin structure at the mating locus [23]. The potential chromatin remodeling function of Mit1 might be involved in facilitating proper positioning of nucleosomes required for the formation of heterochromatin.

\section{Functions of SWI/SNF family proteins in heterochromatin}

\subsection{ATRX}

Human ATRX ( $\alpha$-thalassemia and mental retardation $\mathrm{X}$-linked) is a transcriptional regulator containing an ATPase/helicase domain similar to those in SWI/SNF family proteins [54]. Mutations in ATRX result in X-linked $\alpha$-thalassemia mental retardation (ATR-X) syndrome [55]. ATRX interacts with transcription co-activator Daxx to form a complex that has the ability to disrupt DNA-histone interaction at the entry/exit site of the nucleosome in vitro, but does not alter nucleosome phasing, which is different from chromatin remodeling by SWI/SNF [25].

ATRX is linked to heterochromatin as it interacts, and colocalization, with HP-1 [26,27] ATRX localizes to both heterochromatin and promyelocytic leukaemia (PML) bodies, which is regulated by its cell cycle-dependent phosphorylation [25,28]. PML bodies are involved in the 
reestablishment of condensed heterochromatin state on pericentromeric satellite DNA [29]. The ADD domain of ATRX binds the N-terminal tail of histone $\mathrm{H} 3$ through two binding pockets, one for unmodified $\mathrm{K} 4$ and the other for di- or tri-methylated K9 [30,31]. This combinatorial recognition of the modification state of $\mathrm{H} 3$ tail is required for ATRX localization in vivo, which is enhanced by a third interaction between ATRX and HP1. These interactions help ATRX complex to target pericentric and telomeric heterochromatin.

The Drosophila homolog of human ATRX (dATRX) was also found to co-localize with HP1a and be involved in heterochromatin function [32]. Mutations in dATRX results in a disruption of heterochromatin-mediated position effect variegation. Of the two isoforms of the dATRX protein, the longer one interacts directly with dHP1a both in vitro and in vivo, and is required for HP1a deposition and the formation of pericentric $\beta$-heterochromatin in vivo $[32,33]$.

\subsection{BRG1}

BRG1, the catalytic subunit of a human SWI/SNF chromatin remodeling complex, was found to specifically coimmunoprecipitates with HP1 $\alpha$ in HeLa cells, and preferentially interacts with HP1 $\alpha$ in vitro [34]. Three BRG1 residues essential for the HP1 $\alpha$-BRG1 interaction are also critical for HP1 $\alpha$-mediated gene silencing. These results suggest that BRG1 modulates the structure of HP1 $1 \alpha$-containing heterochromatin [34].

\section{Summary and outlook}

The studies discussed in this article demonstrate an involvement of chromatin remodeling factors in heterochromatin. Chromatin remodelers may act in nucleosome assembly and/or rearrangement during the formation of heterochromatin, or serve to stabilize/maintain heterochromatin structure. Chromatin remodelers are often found to associate with other proteins to form complexes that are targeted to heterochromatin. These proteins include chromatin modifying enzymes such as HDACs that participate in creating heterochromatin-specific chromatin marks, as well as chromatin-binding factors such as HP1 that help direct the chromatin modifying and remodeling enzymes to heterochromatin loci.

Several lines of evidence point to a key role of chromatin remodelers in the replication of heterochromatin. Chromatin remodeling may assist the passage of the DNA replication machinery by decondensing heterochromatin and/or moving nucleosomes to expose nucleosomal DNA in front of the DNA polymerase, or remodel/reposition nascent nucleosomes to facilitate the deacetylation and heterochromatin-specific modifications of histones that are required for the re-establishment of heterochromatin behind the replica- tion machinery. Other data suggest a role of chromatin remodeling in maintaining the stability of heterochromatin. Given the dynamic nature of heterochromatin, it is likely that regions of preexisting heterochromatin may be disrupted and converted to euchromatin by stochastic actions of certain chromatin modifying activities during cell proliferation. Chromatin remodeling may be required to offset such disruptions, and restore heterochromatic characteristics, thereby maintaining the stability of heterochromatin structure.

Detailed molecular mechanisms underlying the functions of chromatin remodeling activities in the establishment and maintenance of heterochromatin have yet to be resolved in future studies. Critical questions to be answered regarding the role of a particular chromatin remodeler in heterochromatin include how it is targeted to heterochromatin, how it cooperates with the silencing machinery and/or other chromatin modifying activities, which activity (e.g., nucleosome sliding, eviction, conformational change, or $\mathrm{H} 2 \mathrm{~A} / \mathrm{H} 2 \mathrm{~B}$ dimer exchange) is actually involved in its function in heterochromatin, as well as whether and how its function is regulated (e.g., by cell cycle progression). Insights into the roles of chromatin remodeling factors in heterochromatin would greatly advance our understanding of the establishment, maintenance and inheritance of heterochromatin.

This work was supported by U.S. National Institutes of Health (Grant No. GM62484).

1 Luger K, Mäder A W, Richmond R K, et al. Crystal structure of the nucleosome core particle at 2.8 A resolution. Nature, 1997, 389: 251-260

2 Grewal S I, Moazed D. Heterochromatin and epigenetic control of gene expression. Science, 2003, 301: 798-802

3 Dillon N. Heterochromatin structure and function. Biol Cell, 2004, 96: 631-637

4 Henikoff S. Heterochromatin function in complex genomes. Biochim Biophys Acta, 2000, 1470: O1-8

5 Rusche L N, Kirchmaier A L, Rine J. The establishment, inheritance, and function of silenced chromatin in Saccharomyces cerevisiae. Annu Rev Biochem, 2003, 72: 481-516

6 Sun F L, Cuaycong M H, Elgin S C. Long-range nucleosome ordering is associated with gene silencing in Drosophila melanogaster pericentric heterochromatin. Mol Cell Biol, 2001, 21: 2867-2879

7 Wallrath L L, Elgin S C. Position effect variegation in Drosophila is associated with an altered chromatin structure. Genes Dev, 1995, 9: 1263-1277

8 Clapier C R, Cairns B R. The biology of chromatin remodeling complexes. Annu Rev Biochem, 2009, 78: 273-304

9 Flaus A, Martin D M, Barton G J, et al. Identification of multiple distinct Snf2 subfamilies with conserved structural motifs. Nucleic Acids Res, 2006, 34: 2887-2905

10 Neves-Costa A, Will W R, Vetter A T, et al. The SNF2-family member Fun30 promotes gene silencing in heterochromatic loci. PLoS ONE, 2009, 4: e8111

11 Yu Q, Zhang X, Bi X. Roles of chromatin remodeling factors in the formation and maintenance of heterochromatin structure. J Biol Chem, 2011, 286: 14659-14669

12 Strålfors A, Walfridsson J, Bhuiyan H, et al. The FUN30 chromatin remodeler, Fft3, protects centromeric and subtelomeric domains from euchromatin formation. PLoS Genet, 2011, 7: e1001334

13 Rowbotham S P, Barki L, Neves-Costa A, et al. Maintenance of si- 
lent chromatin through replication requires SWI/SNF-like chromatin remodeler SMARCAD1. Mol Cell, 2011, 42: 285-296

14 Cuperus G, Shore D. Restoration of silencing in Saccharomyces cerevisiae by tethering of a novel Sir2-interacting protein, Esc8. Genetics, 2002, 162: 633-645

15 Stephens G E, Xiao H, Lankenau D H, et al. Heterochromatin protein 2 interacts with Nap-1 and NURF: a link between heterochromatin-induced gene silencing and the chromatin remodeling machinery in Drosophila. Biochemistry, 2006, 19; 45: 14990-14999

16 Ishimi Y, Kojima M, Yamada M, et al. Binding mode of nucleosome-assembly protein (AP-I) and histones. Eur J Biochem, 1987, 162: 19-24

17 Bozhenok L, Wade P A, Varga-Weisz P. WSTF-ISWI chromatin remodeling complex targets heterochromatic replication foci. EMBO J, 2002, 21: 2231-2241

18 Poot R A, Bozhenok L, van den Berg D L, et al. The Williams syndrome transcription factor interacts with PCNA to target chromatin remodelling by ISWI to replication foci. Nat Cell Biol, 2004, 6: 1236-1244

19 Poot R A, Bozhenok L, van den Berg D L, et al. Chromatin remodeling by WSTF-ISWI at the replication site: opening a window of opportunity for epigenetic inheritance? Cell Cycle, 2005, 4: 543-546

20 Collins N, Poot R A, Kukimoto I, et al. An ACF1-ISWI chromatin-remodeling complex is required for DNA replication through heterochromatin. Nat Genet, 2002, 32: 627-632

21 Kim J, Sif S, Jones B, et al. Ikaros DNA-binding proteins direct formation of chromatin remodeling complexes in lymphocytes. Immunity, 1999, 10: 345-355

22 Helbling Chadwick L, Chadwick B P, Jaye D L, et al. The Mi-2/NuRD complex associates with pericentromeric heterochromatin during $\mathrm{S}$ phase in rapidly proliferating lymphoid cells. Chromosoma, 2009, 118: 445-457

23 Sugiyama T, Cam H P, Sugiyama R, et al. SHREC, an effector complex for heterochromatic transcriptional silencing. Cell, 2007, 128: 491-504

24 Motamedi M R, Hong E J, Li X, et al. HP1 proteins form distinct complexes and mediate heterochromatic gene silencing by nonoverlapping mechanisms. Mol Cell, 2008, 32: 778-790

25 Xue Y, Gibbons R, Yan Z, et al. The ATRX syndrome protein forms a chromatin-remodeling complex with Daxx and localizes in promyelocytic leukemia nuclear bodies. Proc Natl Acad Sci USA, 2003, 100: $10635-10640$

26 Lechner M S, Schultz D C, Negorev D, et al. The mammalian heterochromatin protein 1 binds diverse nuclear proteins through a common motif that targets the chromoshadow domain. Biochem Biophys Res Commun, 2005, 331: 929-937

27 McDowell T L, Gibbons R J, Sutherland H, et al. Localization of a putative transcriptional regulator (ATRX) at pericentromeric heterochromatin and the short arms of acrocentric chromosomes. Proc Natl Acad Sci USA, 1999, 96: 13983-13988

28 Ishov A M, Vladimirova O V, Maul G G. Heterochromatin and ND10 are cell-cycle regulated and phosphorylation-dependent alternate nuclear sites of the transcription repressor Daxx and SWI/SNF protein ATRX. J Cell Sci, 2004, 117: 3807-3820

29 Luciani J J, Depetris D, Usson Y, et al. PML nuclear bodies are highly organised DNA-protein structures with a function in heterochromatin remodelling at the G2 phase. J Cell Sci, 119: 2518-2531

30 Eustermann S, Yang J C, Law M J, et al. Combinatorial readout of histone $\mathrm{H} 3$ modifications specifies localization of ATRX to heterochromatin. Nat Struct Mol Biol, 2011, 18: 777-7782

31 Dhayalan A, Tamas R, Bock I, et al. The ATRX-ADD domain binds to $\mathrm{H} 3$ tail peptides and reads the combined methylation state of $\mathrm{K} 4$ and K9. Hum Mol Genet, 2011, 20: 2195-2203

32 Bassett A R, Cooper S E, Ragab A, et al. The chromatin remodelling factor dATRX is involved in heterochromatin formation. PLOS ONE, 2008, 3: e2099

33 Emelyanov A V, Konev A Y, Vershilova E, et al. Protein complex of
Drosophila ATRX/XNP and HP1a is required for the formation of pericentric beta-heterochromatin in vivo. J Biol Chem, 2010, 285: 15027-15037

34 Nielsen A L, Sanchez C, Ichinose H, et al. Selective interaction between the chromatin-remodeling factor BRG1 and the heterochromatin-associated protein HP1alpha. EMBO J, 2002, 21: 5797-5806

$35 \mathrm{Ng} \mathrm{H} \mathrm{H}$, Ciccone D N, Morshead K B, et al. Lysine-79 of histone H3 is hypomethylated at silenced loci in yeast and mammalian cells: a potential mechanism for position-effect variegation. Proc Natl Acad Sci USA, 2003, 100: 1820-1825

36 Santos-Rosa H, Bannister A J, Dehe P M, et al. Methylation of H3 lysine 4 at euchromatin promotes Sir3p association with heterochromatin. J Biol Chem, 2004, 279: 47506-47512

37 Moazed D. Enzymatic activities of Sir2 and chromatin silencing. Curr Opin Cell Biol, 2001, 13: 232-238

38 Weiss K, Simpson R T. High-resolution structural analysis of chromatin at specific loci: Saccharomyces cerevisiae silent mating type locus HMLalpha. Mol Cell Biol, 1998, 18: 5392-5403

39 Ravindra A, Weiss K, Simpson R T. High-resolution structural analysis of chromatin at specific loci: Saccharomyces cerevisiae silent mating-type locus HMRa. Mol Cell Biol, 1999, 19: 7944-7950

40 Loney E R, Inglis P W, Sharp S, et al. Repressive and non-repressive chromatin at native telomeres in Saccharomyces cerevisiae. Epigenetics Chromatin, 2009, 2: 18

41 Awad S, Ryan D, Prochasson P, et al. The Snf2-homolog Fun30 acts as a homodimeric ATP-dependent chromatin-remodeling enzyme. J Biol Chem, 2010, 285: 9477-9484

42 Hurley J H, Lee S, Prag G. Ubiquitin-binding domains. Biochem J, 2006, 399: 361-372

43 Udvardy A, Maine E, Schedl P. The 87A7 chromomere. Identification of novel chromatin structures flanking the heat shock locus that may define the boundaries of higher order domains. J Mol Biol, 1985, 185: $341-358$

44 Chung J H, Bell A C, Felsenfeld G. Characterization of the chicken beta-globin insulator. Proc Natl Acad Sci USA, 1997, 94: 575-580

45 Dhillon N, Raab J, Guzzo J, et al. DNA polymerase epsilon, acetylases and remodellers cooperate to form a specialized chromatin structure at a tRNA insulator. EMBO J, 2009, 28: 2583-2600

46 Bi X, Yu Q, Sandmeier J J, et al. Formation of boundaries of transcriptionally silent chromatin by nucleosome-excluding structures. Mol Cell Biol, 2004, 24: 2118-2131

47 Oki M, Kamakaka R T. Barrier function at HMR. Mol Cell, 2005, 19: 707-716

48 Felsenfeld G, Groudine M. Controlling the double helix. Nature, 2003, 421: 448-453

49 Rando O J, Verstrepen K J. Timescales of genetic and epigenetic inheritance. Cell, 2007, 128: 655-668

50 Corona D F, Tamkun J W. Multiple roles for ISWI in transcription, chromosome organization and DNA replication. Biochim Biophys Acta, 2004, 1677: 113-119

51 Mellor J, Morillon A. ISWI complexes in Saccharomyces cerevisiae. Biochim Biophys Acta, 2004, 1677: 100-112

52 Corona D F, Siriaco G, Armstrong J A, et al. ISWI regulates higher-order chromatin structure and histone $\mathrm{H} 1$ assembly in vivo. PLoS Biol, 2007, 5: e232

53 Badenhorst P, Voas M, Rebay I, et al. Biological functions of the ISWI chromatin remodeling complex NURF. Genes Dev, 2002, 16: 3186-3198

54 Picketts D J, Higgs D R, Bachoo S, et al. ATRX encodes a novel member of the SNF2 family of proteins: mutations point to a common mechanism underlying the ATR-X syndrome. Hum Mol Genet, 1996, 5: 1899-1907

55 Gibbons R J, Picketts D J, Villard L, et al. Mutations in a putative global transcriptional regulator cause X-linked mental retardation with alpha-thalassemia (ATR-X syndrome). Cell, 1995, 80: 837845 


\section{Biographical Sketch}

Dr. Bi Xin is an Associate Professor in the Department of Biology at University of Rochester, Rochester, NY, USA. He obtained a Bachelor of Science degree in molecular biology from the University of Science and Technology of China in 1988. As a CUSBEA fellow, Dr. Bi joined the graduate program at Johns Hopkins University School of Medicine, Baltimore, MD, USA in 1989. He earned a Ph.D. degree in biological chemistry in 1994. Dr. Bi then trained as a post-doctoral fellow at Princeton University, before joining the Biochemistry Department of University of Nebraska-Lincoln as an Assistant Professor in 2000. He moved to University of Rochester in 2003. His lab is focused on understanding the molecular mechanisms underlying the structure and function of heterochromatin in the yeast model organism. Dr. Bi teaches the upper level undergraduate (and graduate) course of Eukaryotic Gene Regulation.

Open Access This article is distributed under the terms of the Creative Commons Attribution License which permits any use, distribution, and reproduction in any medium, provided the original author(s) and source are credited. 